\title{
Experimental study of the transient motion of floats reproducing floating wood in rivers
}

\author{
Hossein Ghaffarian Roohparvar, ${ }^{1, *}$, Diego Lopez ${ }^{1}$, Nicolas Riviere ${ }^{1}$, Herve Piegay ${ }^{2}$, \\ and Emmanuel Mignot ${ }^{1}$ \\ ${ }^{1}$ Univ. of Lyon, INSA Lyon, CNRS, LMFA, F-69100 Villeurbanne, France \\ ${ }^{2}$ Univ. of Lyon, UMR 5600, Environnement-Ville-Société CNRS, F-69362 Lyon, France
}

\begin{abstract}
The flow of large wood among hydraulic structures in rivers, especially in urban areas, can cause many problems. Despite many statistical, morphological and hydrodynamical studies on this phenomenon, little information is available on the transient motion of floating wood pieces. In this study, we investigate theoretically and experimentally the transient motion of floating particles under a simple acceleration. From a standard advection model we identify a particle characteristic response distance to the flow, noted $\lambda$. This key parameter is then measured for different floating particles reproducing wood in rivers (logs without and with idealized roots). We show here the typical value of this parameter as a function of particle streamwise body length for different particle geometries. The influence of roots can be well captured by an equivalent frontal area, regardless of the root pattern. This response distance could provide useful information on the probability of impact on hydraulic structures depending on the floating wood characteristics.
\end{abstract}

\section{Introduction}

Floating wood in waterways provides a rich source of nutrients and a suitable habitat for different biological species [1]. Therefore, the presence of large wood along the river's path is positive and even necessary. However, the presence of floating wood also can lead to log jam in some sections of the river, reducing the channel cross-section area, hence increasing the water level and hydrostatic force on the hydraulic structure to which the jam is attached [2]. This is even more critical during exceptional floods, as it increases the risk of flooding and damage, in particular through the destruction of jam breakage [3, 4]. Due to the concentration of various hydraulic structures such as bridges, dikes, and diversion systems along the rivers and especially in urban areas, the study of wood dynamics in river is crucial in order to reduce the flooding risk and maintenance costs for hydraulic structures and urban facilities along the rivers.

In general, mobility of large wood in rivers is unfrequent and only increases when large floods occur [4]. Gurnell et al. [5] showed that the relationship between tree length and river width can affect the dynamics of large woods and log jams along the river. They showed that while in large channels the flow regime has a dominant effect on wood mobility, wood length

\footnotetext{
*e-mail: hossein.ghaffarian@insa-lyon.fr
} 
and channel width play a significant role in small and medium channels. Ruiz-Villanueva et al. [6] provided comprehensive information on factors affecting trunk transport along rivers including river morphology and hydrology, floating particle geometry, as well as data on the transfer of wood in a flood event. They showed that in shallow flows, the diameter of the particle has a major effect, while in deep flow wood length is a key factor in wood transportation. Additionally they observed that the distance travelled by large wood decreases sharply as the particle volume increases. Wood dimensions have also been shown to play a significant role in log jam formation, whose frequency increases with increasing wood length and decreasing diameter.

In order to predict floating wood transport, many studies focused on numerical modelling of wood dynamics, relying on semi-empirical models and theoretical advection models [7-12]. These models benefit from experimental measures of the drag force on floating particles $[11,13]$. In a recent study, Persi et al. [12] compared the trajectory of floating logs simulated with a theoretical advection model with previous experiments and simulations of Ruiz-Villanueva et al. [9], showing a very good agreement and providing a promising theoretical tool for predicting wood transport in rivers. However, despite the recent interest in modelling floating wood in rivers, the generalisation of these results for different types of floats and flow configurations is yet difficult to achieve, as there is no general characterization of floating particles dynamics under flow, in particular considering their transient motion.

In this work, we study theoretically and experimentally the transient dynamics of floating particles representing wood in rivers, considering the simple case of acceleration under a uniform one-dimensional flow. After introducing the advection model used for predicting floating particles acceleration in Sec. 2, we present experimental setup and tested floating particles in Sec 3. We then present and discuss our results in Sec. 4.

\section{Advection model}

The motion of a floating particle under flow can be modelled by a standard advection equation based on Newton's second law,

$$
m_{p} \frac{d \mathbf{v}}{d t}=\mathbf{F}_{D}+\mathbf{F}_{L}+\mathbf{F}_{A}
$$

where $m_{p}$ is the particle mass, $\mathbf{v}$ its velocity, $t$ is the time, and $\mathbf{F}_{D}, \mathbf{F}_{L}$ and $\mathbf{F}_{A}$ denote respectively the drag, lift (or lateral) and added mass forces [10-12, 14, 15]. In the general case, for inertial particles for which the drag and lift forces are quadratic with the relative velocity, the expression of these forces is not well known and requires specific attention depending on the object considered. As we focus in this study on symmetric objects with respect to the flow axis, there is no lift force, and Eq. (1) can be written as

$$
\left(m_{p}+\frac{1}{2} C_{a} m_{f}\right) \frac{d \mathbf{v}}{d t}=\frac{1}{2} \rho_{f} C_{d} S|\mathbf{u}-\mathbf{v}|(\mathbf{u}-\mathbf{v})+m_{f}\left(1+\frac{1}{2} C_{a}\right) \frac{D \mathbf{u}}{D t},
$$

where $m_{f}$ is the equivalent fluid mass which is equal to $m_{p}$ for a floating object, $\mathbf{u}$ the fluid velocity, $C_{d}$ and $C_{a}$ are respectively the drag and added mass coefficients and $S$ is a reference frontal area; $D / D t$ denotes the derivative following the fluid element [12].

This equation can be expressed in a non-dimensional form using typical flow scales $L_{0}$ and $U_{0}$ as reference scales, resulting in

$$
\frac{d \mathbf{v}^{\star}}{d t^{\star}}=\frac{L_{0}}{\lambda}\left|\mathbf{u}^{\star}-\mathbf{v}^{\star}\right|\left(\mathbf{u}^{\star}-\mathbf{v}^{\star}\right)+\frac{D \mathbf{u}^{\star}}{D t^{\star}},
$$


where dimensionless quantities are denoted by a star, and

$$
\lambda=2 \frac{1+C_{a}}{C_{d}} \frac{\rho_{p}}{\rho_{f}} \frac{V_{p}}{S},
$$

$V_{p}$ being the particle volume, and $\rho_{p}$ and $\rho_{f}$ the particle and fluid densities. In this equation, $\lambda$ is a characteristic length that depends only on the object considered and the fluid density, but not on the flow. Indeed for objects whose Reynolds number is high (typically larger than $10^{3}$ ) such as floating wood in rivers, the drag and added mass coefficients can be considered to be constant.

An interpretation of the parameter $\lambda$ can be obtained from Eq. (3), by analogy with the Stokes number defined for small particles at low inertia (see for instance [16]). When $\lambda$ is small relative to the flow length scale $L_{0}$, Eq. (3) results in $\mathbf{v}^{\star} \cong \mathbf{u}^{\star}$, so that the floating particle acts as a flow tracer. Conversely when $\lambda \gg L_{0}$, the floating particle is not affected by the flow and its trajectory diverges from the flow trajectory. In the case of wood transported by a river approaching to an obstacle like a bridge pier, the probability of collision therefore increases with $\lambda$. This length can thus be seen as a typical response distance to the flow and is referred in the following to the particle characteristic response distance.

It is interesting to note that Eq. (3) simplifies greatly if we consider the acceleration of a particle initially at rest under a uniform steady flow. In that case, as there is no flow length scale, one can choose $L_{0}=\lambda$ for normalization, and the advection equation can be simplified into the following scalar equation

$$
\frac{d v^{\star}}{d t^{\star}}=\left(1-v^{\star}\right)^{2}
$$

where $v^{\star}$ is the streamwise particle velocity normalized by the surface flow velocity $U_{0}$ and $t^{\star}$ is the time normalized by $\lambda / U_{0}$. This equation can be integrated analytically so that the non-dimensional velocity $v^{\star}$ and position $x^{\star}$ read

$$
v^{\star}\left(t^{\star}\right)=1-\frac{1-v_{0}^{\star}}{1+\left(1-v_{0}^{\star}\right) t^{\star}}, \quad x^{\star}\left(t^{\star}\right)=t^{\star}-\ln \left[1+\left(1-v_{0}^{\star}\right) t^{\star}\right]
$$

considering that $v^{\star}\left(t^{\star}=0\right)=v_{0}^{\star}$ and $x^{\star}\left(t^{\star}=0\right)=0$. As a result, the characteristic response distance $\lambda$ of any floating particles can be determined by its acceleration under a uniform one-dimensional flow.

\section{Methods}

In order to measure the characteristic response distance of different floating particles, we perform an experimental study of particle acceleration under a uniform and steady flow, whose theoretical solution is given in Eq. (6).

\subsection{Flow}

The experiments are carried out in a straight rectangular $8 \mathrm{~m}$ long and $0.25 \mathrm{~m}$ wide flume. In order to control the flow discharge, the flume is equipped with a control valve and an electromagnetic flow-meter, and an adjustable weir at the end of the flume controls the water level. The experiments are performed with a steady, turbulent and subcritical flow. Surface velocity measured by Particle tracking velocimetry (PTV) technique, using small floating papers as tracer. 


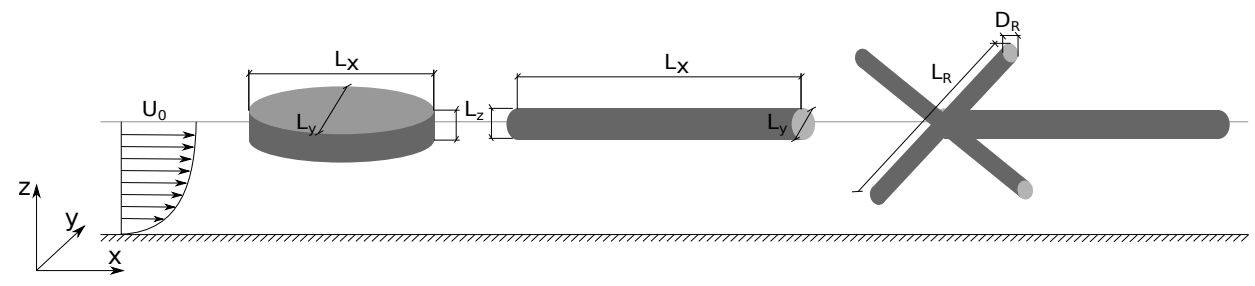

Figure 1. Schematic representation of particle geometries and flow with corresponding parameters.

\subsection{Floating particles}

Two categories of floating particles with increasing complexity are tested in the experiments (Fig. 1). The first category consists in paraffin disks $\left(\rho_{p} / \rho_{f}=0.88\right)$ with fixed height $\left(L_{z}=\right.$ $1 \mathrm{~cm})$ and different diameters. A first advantage presented by a disk is its isotropy, so that the orientation with respect to the flow does not affect its dynamics. Secondly, a disc with small height will minimize the effect of vertical velocity profile, the particle will be only subjected to the surface velocity of the flow. Finally, the contact angle between paraffin and water is close to 90 degrees which reduces the effect of surface tension on the particle movement. The second category of floating particles are simple cylindrical wooden logs $\left(\rho_{p} / \rho_{f}=0.63\right)$, with or without idealized roots, which mimic floating wood in rivers. For simplification, the effect of orientation is not addressed here and all logs are tested aligned with the streamwise direction.

The parametrization is described in Fig. 1. The flow surface velocity is noted $U_{0}$ along the $x$ direction and is assumed to be the same over the immersed depth of the different objects. The different objects are characterized by their streamwise, spanwise and vertical dimensions $L_{x}, L_{y}$ and $L_{z}$ respectively, and we define the aspect ratio $A=L_{z} / L_{x}$. The aspect ratio ranges from 0.15 to 0.8 for disks and 0.05 to 0.9 for logs. Considering the logs with roots, all logs have the same aspect ratio $L_{z} / L_{x}=0.1$, and the root-wad is characterized by the number of roots $N_{R}\left(N_{R}=2\right.$ in Fig. 1), the rood diameter $D_{R}$ and total length $L_{R}$. In the rooted $\log$ experiments $N_{R}=1$ to $4, D_{R} / L_{z}=0.4$ and $L_{R} / L_{z}=3.5$ to 15 . For all the particles tested the vertical dimension $L_{z}$ was kept constant and equal to $1 \mathrm{~cm}$, and the typical particle Reynolds numbers are comprised between $10^{3}$ and $10^{4}$, so that the assumption of constant drag coefficient is valid and Eq. (6) should be satisfied [17].

By defining particle Froude and Reynolds numbers as,

$$
F r_{p}=\frac{U_{0}}{\sqrt{g D_{h}}} \quad, \quad R e_{p}=\frac{\rho_{f}\left(U_{0}-v\right) D_{h}}{\mu}
$$

where $D_{h}$ is particle submerged hydraulic diameter, in natural rivers, the particle Froude number values range from 0.5 to 1 and the particle Reynolds number between $10^{4}$ and $5 \times 10^{4}$ for floating wood [6]. In the experiments the particle Froude number is around 0.8 and the particles Reynolds numbers are around $10^{3}$.

\subsection{Measure of the characteristic response distance}

The particle trajectories are recorded using a high spatial resolution camera JAI GO-5000M $\left(2560 \times 1700 \mathrm{px}^{2}\right)$ at 60 frames per second. The camera was located above the flume, perpendicular to the flow. The time evolution of the particle position is determined with a standard image detection algorithm using Matlab R2017a ${ }^{\circledR}$. Then, the model equation Eq. (6) is fitted 
(a)

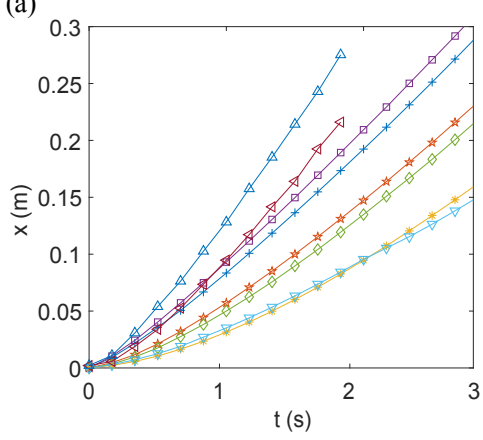

(b)

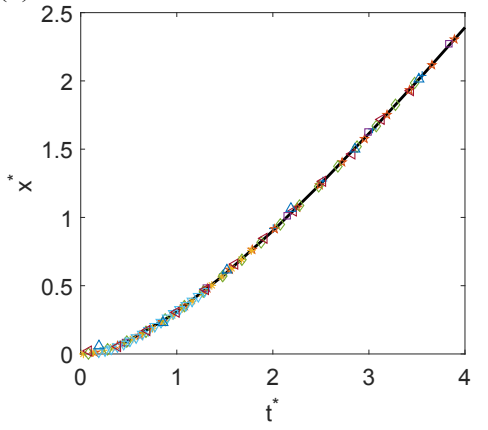

+ Disk, $\mathrm{L}_{\mathrm{x}}=20 \mathrm{~mm}$

Disk, $L_{x}=32 \mathrm{~mm}$

Disk, $L_{x}=63 \mathrm{~mm}$

Log, $\mathrm{L}_{x}=22 \mathrm{~mm}$

$\log , \mathrm{L}_{\mathrm{x}}=51 \mathrm{~mm}$

$\log , L_{x}=210 \mathrm{~mm}$

Rooted log, $\mathrm{L}_{\mathrm{R}}=35 \mathrm{~mm}$

Rooted log, $\mathrm{L}_{R}=150 \mathrm{~mm}$

$1 \mathrm{D}$ advection model

Figure 2. a) Streamwise position as a function of time for different floating particles; b) Same trajectories normalized by the particle characteristic response distance $\lambda$ as a function of the normalized time $t^{\star}=\lambda t / U_{0}$, and $1 \mathrm{D}$ advection model of Eq. (8).

on the experimental trajectory by a root mean square method, using only $\lambda$ as fitting parameter.

\section{Results}

\subsection{Model validation}

We first validate the one-dimensional advection model by comparing different experiments with the theoretical model of Eq. (6). Fig. 2a shows several trajectories recorded for different particles. Then on Fig. $2 b$ we plot the normalized trajectories, which collapse all onto the advection model (black line). Note that due to the different initial velocities $v_{0}$, the normalized trajectory were shifted in time so that $v^{\star}\left(t^{\star}=0\right)=0$. As a result the theoretical trajectory is given by

$$
x^{\star}\left(t^{\star}\right)=t^{\star}-\ln \left(1+t^{\star}\right) .
$$

In this study, tests for 13 different particle geometries are repeated 10 times, with coefficients of determination of more than 0.999 in almost all cases. Based on Fig. 2b, it can be concluded that the model matches perfectly well with experimental data. It can also be concluded that $\lambda$ is a good parameter for interpreting the transient motion of a floating particle.

\subsection{Particle aspect ratio}

In this section, the effect of changing the particle aspect ratio $A$ for disks and logs is presented. Six different disks and seven different logs were tested. Fig. 3 presents the particle characteristic response distance $\lambda$ normalized by the streamwise body length $L_{x}$ as a function of particle aspect ratio $\left(A=L_{z} / L_{x}\right)$. The error bars show the standard deviation of the repetitions.

We readily see that typical particle characteristic response distances are about two body lengths of the floating particles. Additionally the graph shows that there exists a maximum value of $\lambda$ for a particular value of $A$ for both disk and log. According to this graph, the peak point for the relative particle characteristic response distance occurs at $A \cong 0.2$. In the case of a floating particle, a large response distance means that the particle travels longer distances 


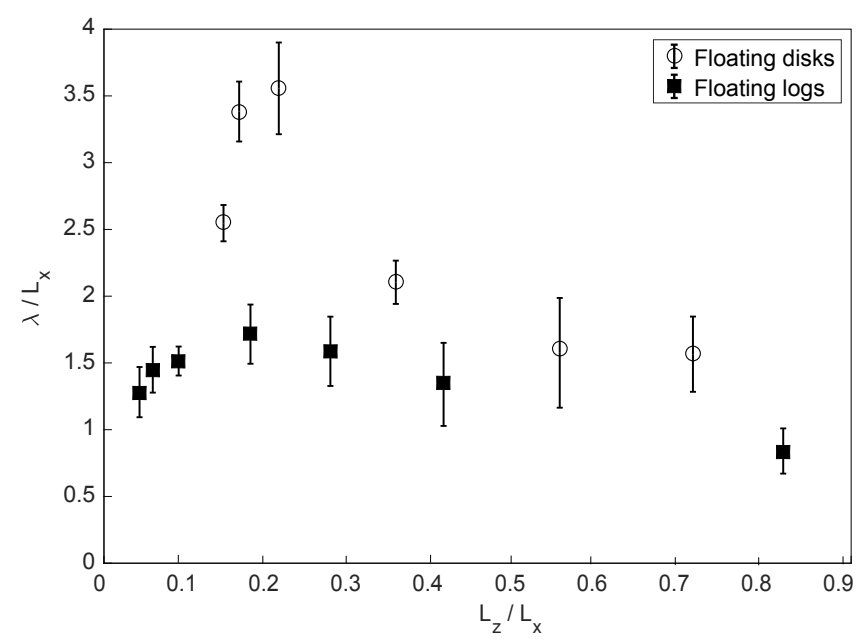

Figure 3. Particle characteristic response distance normalized by the streamwise body length $\left(\lambda / L_{x}\right)$ as a function of particle aspect ratio $\left(A=L_{z} / L_{x}\right)$ for floating disks (dashed line) and logs (solid line).

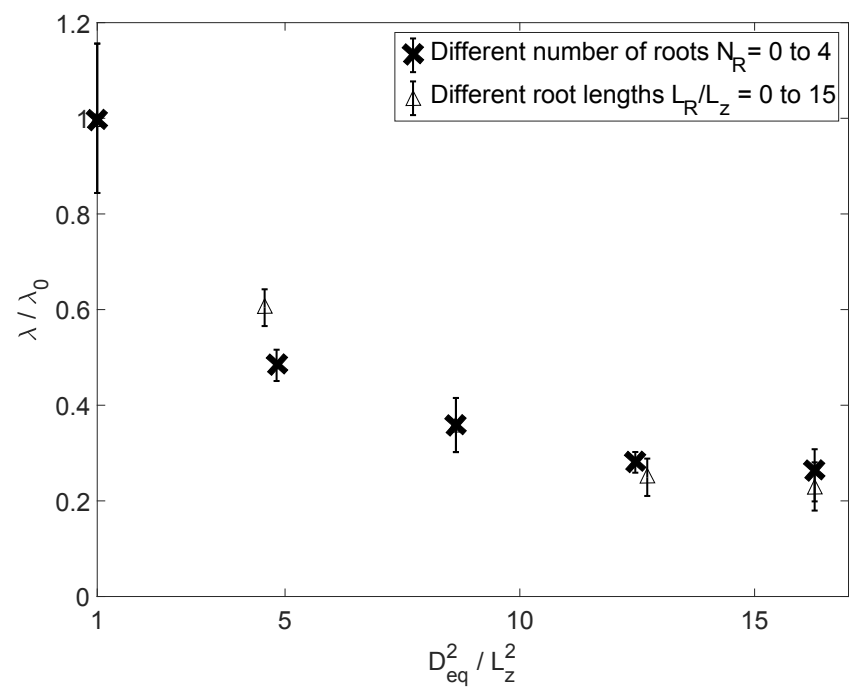

Figure 4. Particle characteristic response distance of a $\log$ with root normalized by its rootless value $\left(\lambda / \lambda_{0}\right)$ as a function of the ratio between root-wad and $\log$ frontal area $D_{e q}^{2} / L_{z}^{2}$ : varying $N_{R}$ from 0 to 4 $\left(L_{R} / L_{z}=7.5\right)$ in dashed line, and varying $L_{R} / L_{z}$ from 0 to $15\left(N_{R}=2\right)$ in solid line.

before adapting to the flow. As discussed in Sec. 2, this maximum value can be seen as a critical value, as it results in a higher probability that the particle hits an obstacle. It should be noted that, although the peak in this graph occurs for both categories of particles at almost the same aspect ratio, the corresponding geometries are quite different for disks and logs. 


\subsection{Influence of roots}

The presence of roots is expected to affect the characteristic response distance of log particles. To check this effect, we test two different parameters, the length and number of roots. In order to have a common representation for the different types of roots we define the equivalent diameter $D_{e q}$ of a rootless log with the same frontal area, which reads

$$
D_{e q}=\sqrt{\frac{4 N_{R} D_{R}\left(L_{R}-L_{z}\right)}{\pi}+L_{z}^{2}},
$$

Hence $D_{e q}^{2} / L_{z}^{2}$ is the ratio between the root-wad and log frontal areas.

Fig. 4 presents the effect of root frontal area on particle characteristic response distance. Here $\lambda$ is normalized by $\lambda_{0}$, the log response distance with same characteristics but without roots. As seen in this figure, by adding roots to a log, its characteristics response distance decreases $\left(\lambda / \lambda_{0}<1\right)$ and this effect increases as the root frontal area increases. In addition, the change in the number of roots $N_{R}$ or the length of the roots $L_{R}$ has the same effect on $\lambda$, and with the chosen representation, the two curves superimposed well. This means that $\lambda$ is function of particle frontal area rather than root pattern. This graph could be a useful tool to measure $\lambda$ for logs with root according to their response distance without roots.

\section{Conclusion}

In this work, considering a standard advection model for floating particles reproducing floating wood in rivers we identified a key physical parameter that drives the floating particle's response to the flow. This parameter is the characteristic response distance noted $\lambda$ that scales the distance travelled by the particle during its transient motion. It is interesting to note that for floating particles such as wood in rivers this distance is independent of the flow velocity. Our experiments show that this distance is about two to three times the streamwise body length. This parameter depends on the object vertical to streamwise aspect ratio and reaches a maximum for an aspect ratio of 0.2 . In the presence of roots, we find that only the resulting frontal area plays a significant role and not the root pattern.

Since $\lambda$ is a particle characteristic that is independent of the flow, these measures made in a uniform flow are expected to remain valid in any flow condition. Our results were obtained on simple symmetric geometries, and we can expect some changes when considering realistic objects. However, the scaling from Eqs. (3) and (4) was obtained in the general case, therefore the results should not be significantly altered, and this approach could be extrapolated to real pieces of wood in natural conditions. The comparison between the object characteristic response distance and typical flow scales (such as a bridge pier size) should provide useful insights on the probability of impact with river infrastructures and associated flooding and damage risk of wood trapping.

\section{Acknowledgement}

This work was performed within the framework and with the support of the LABEX IMU (ANR-10-LABX-0088) of Université de Lyon, within the program "Investissements d'Avenir" (ANR-11-IDEX-0007) operated by the French National Research Agency (ANR).

\section{References}

[1] T.J. Beechie, T.H. Sibley, Transactions of the American Fisheries Society 126, 217 (1997) 
[2] P. De Cicco, E. Paris, L. Solari, River Flow2016 pp. 2341-2345 (2016)

[3] D. Allen, S. Arthur, H. Haynes, S.G. Wallis, N. Wallerstein et al., Science ChinaTechnological Sciences 57, 1512 (2014)

[4] P. Mikuś, B. Wyżga, V. Ruiz-Villanueva, J. Zawiejska, R.J. Kaczka, M. Stoffel, in Flood Risk in the Upper Vistula Basin (Springer, 2016), pp. 77-101

[5] A. Gurnell, H. Piegay, F. Swanson, S. Gregory, Freshwater Biology 47, 601 (2002)

[6] V. Ruiz-Villanueva, B. Wyżga, J. Zawiejska, M. Hajdukiewicz, M. Stoffel, Geomorphology 272, 21 (2016)

[7] C.A. Braudrick, G.E. Grant, Water resources research 36, 571 (2000)

[8] C. Yin, L. Rosendahl, S.K. Kær, H. Sørensen, Chemical Engineering Science 58, 3489 (2003)

[9] V. Ruiz-Villanueva, E. Bladé, M. Sánchez-Juny, B. Marti-Cardona, A. Díez-Herrero, J.M. Bodoque, Journal of Hydroinformatics 16, 1077 (2014)

[10] B. Mazzorana, J. Hübl, A. Zischg, A. Largiader, Natural Hazards 56, 425 (2011)

[11] R. Gonçalves, G. Franzini, G. Rosetti, J. Meneghini, A. Fujarra, Journal of Fluids and Structures 54, 122 (2015)

[12] E. Persi, G. Petaccia, S. Sibilla, Natural Hazards pp. 1-16 (2017)

[13] K. Klaka, J. Penrose, R. Horsley, M. Renilson, Experimental Thermal and Fluid science 30, 131 (2005)

[14] J. Chaplin, P. Teigen, Journal of Fluids and Structures 18, 271 (2003)

[15] M. Benitz, D. Carlson, B. Seyed-Aghazadeh, Y. Modarres-Sadeghi, M. Lackner, D. Schmidt, Computers \& Fluids 136, 247 (2016)

[16] M.R. Maxey, Physics of Fluids 30, 1915 (1987)

[17] S.F. Hoerner, Fluid-dynamic drag: practical information on aerodynamic drag and hydrodynamic resistance (Hoerner Fluid Dynamics, 1965) 